\title{
Improving Brain-Computer Interfaces Using Independent Component Analysis
}

\author{
Yijun Wang ${ }^{1}$, Tzyy-Ping Jung ${ }^{1,2}$ \\ 1 Institute for Neural Computation, University of California San Diego, San Diego, USA \\ 2 Institute of Engineering in Medicine, University of California San Diego, San Diego, USA
}

\section{Introduction}

In the past two decades, electroencephalogram (EEG)-based brain-computer interfaces (BCIs) have attracted much attention in the fields of neuroscience and neural engineering [1-3]. Researchers have made significant progress in designing and demonstrating usable BCI systems for the purpose of communication and control. Currently, the BCI community puts great effort into translating this technology from laboratory demonstrations to real-life products to help physically disabled people achieve improved quality of life [4-5]. Although many studies have been carried out to implement and evaluate demonstration systems in laboratory settings, developing practical BCI systems within a real-world environment still poses severe technical challenges.

In real-world applications, a BCI system must meet the requirements of convenient system use as well as robust system performance [6]. Recently, researchers have proposed different methods for improving the practicality of a BCI system in terms of hardware and software design. When working with a BCI product, researchers need to pay attention to two major issues: (1) ease-of-use, and (2) robustness of system performance. Current BCI research places increasing demand on advanced signal-processing techniques to improve system performance and ease-of-use. Among the different signal-processing techniques employed in current BCI systems, independent component analysis (ICA) is one of the most successful methods [7]. Due to its capability in decomposing scalp EEG signals into functionally independent brain activities and other non-neural activities, ICA has been widely applied to improve the signal-to-noise ratio (SNR) of task-related EEG signals in BCI systems. This study focuses on the use of ICA in current BCI systems. The goal of this study is twofold: (1) to investigate the feasibility of using ICA to improve BCI performance through reviewing the state-of-the-art BCI studies; (2) to introduce our recent work on developing an ICA-based zerotraining method for deriving EEG spatial filters in a motor imagery-based BCI. This study applied the extended infomax ICA algorithm [8] from the open-source EEGLAB toolbox [9] to multichannel EEG data. 


\section{ICA in EEG signal processing}

Independent component analysis is a statistical method that aims to find linear projections of the observed data that maximize their mutual independence [10]. When applied to blind source separation (BSS), ICA aims to recover independent sources using multi-channel observations of mixtures of those sources. In the past two decades, ICA has been successfully used in processing biomedical signals including EEG, electrocardiogram (ECG), magnetoencephalogram (MEG), and functional magnetic resonance imaging (fMRI) signals [11]. In EEG signal processing, ICA has shown a good capability in separating the scalp EEG signals into functionally independent sources, such as neural components originating from different brain areas and artifactual components attributed to eye movements, blinks, muscle, heart, and line noise (Fig. 1). Due to its superiority in EEG source separation, ICA has been successfully applied to EEG research to reduce EEG artifact, enhance the SNR of task-related EEG signals, and facilitate EEG source localization [12-15].

Given a linear mixing model, $n$-channel scalp EEG signals, $\boldsymbol{x}=\left[x_{1} x_{2} \ldots x_{n}\right]$ are generated by $m$ independent sources $\boldsymbol{S}=\left[\begin{array}{llll}s_{1} & s_{2} & \ldots & s_{m}\end{array}\right]$ :

$$
\boldsymbol{x}=\mathbf{A s}
$$

where $\mathbf{A}$ is the $n \times m$ mixing matrix in the model. After ICA, recovered source signals, $\boldsymbol{u}$, can be estimated by applying an unmixing matrix $\mathbf{W}(n \times m)$ to the observed EEG data $\boldsymbol{x}$ :

$$
\boldsymbol{u}=\mathbf{W} \boldsymbol{x} \quad \boldsymbol{x}=\mathbf{W}^{-1} \boldsymbol{u}
$$

where each row of $\mathbf{W}$ is a spatial filter for estimating an independent component (IC) and each column of $\mathbf{W}^{-1}$ consists of electrode weights (i.e., a spatial projection) of an independent component.

Fig. 1 shows an example of ICA applied to 128-channel scalp EEG data recorded during a visually guided reaching task, which involved various kinds of movement artifacts [16]. It is apparently difficult to read the underlying neural activities from the scalp channel data, which include overlapped EEG signals and artifacts. For example, electrodes at the frontal area have very strong eye-movement artifacts, which seriously contaminated the midline theta activities over the prefrontal cortex area. In this example, ICA successfully separates scalp EEG signals into neural and non-neural independent source activities, which can be easily understood according to their spatio-temporal characteristics. As shown in Fig. 1, recovered independent brain activities include the left/right mu components over the sensorimotor areas (L-mu, R-mu), the midline prefrontal component (MPFC), and the posterior parietal components (MPPC, LPPC, RPPC). In addition, ICA also recovered the non-neural source activities including the vertical/horizontal Electrooculogram (VEOG/HEOG), ECG, and Electromyogram (EMG) components. This capability of decomposing scalp EEG signals into functionally independent sources makes ICA a potential tool for many applications in EEG-based BCIs. 

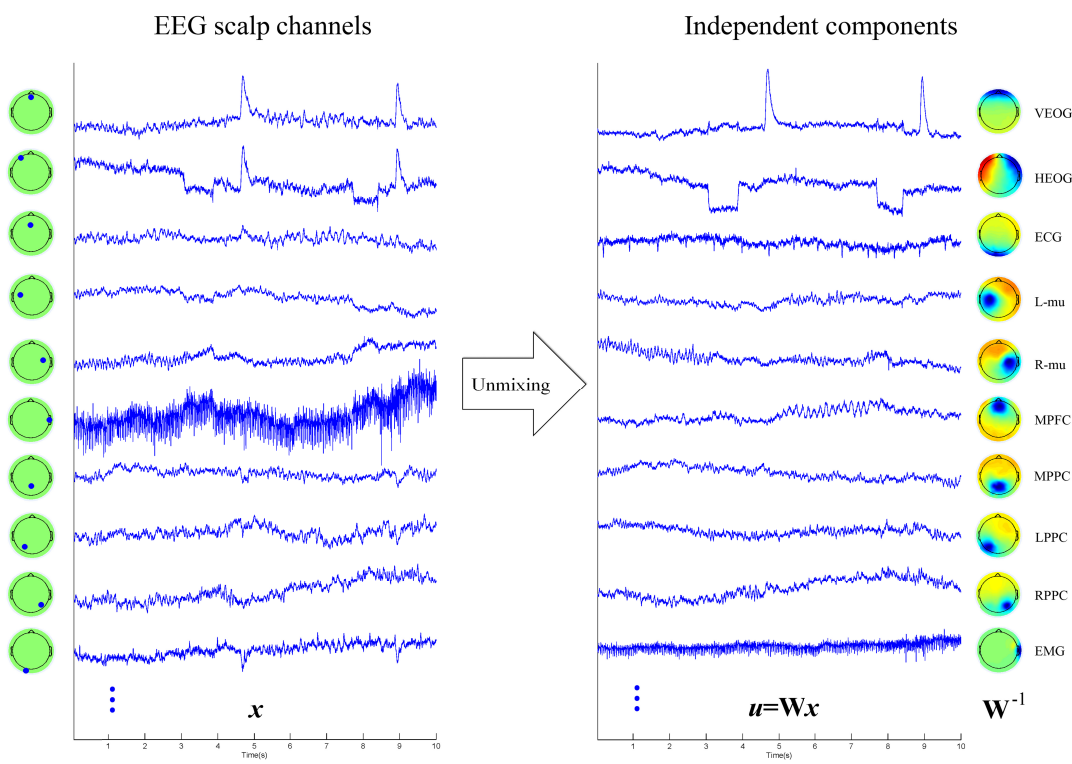

Fig. 1. Schematic overview of ICA decomposition of scalp EEG data $(\boldsymbol{x})$. Activities of independent components $(\boldsymbol{u})$, were obtained by applying an unmixing matrix $\mathbf{W}$ to $\boldsymbol{x}: \boldsymbol{u}=\mathbf{W} \boldsymbol{x}$. Each column of $\mathbf{W}^{-1}$, which consists of electrode weights, was shown as a scalp map and referred to as the spatial pattern of an IC. The spatial patterns $\left(\mathbf{W}^{-1}\right)$ clearly showed scalp distributions of source activities of the ICs.

\section{ICA in BCI systems}

To better understand the state-of-the-art of ICA in BCI studies, this study first presents a survey of the literature. The articles selected for this survey were chosen from journal and conference research papers found in Google scholar using the following keywords: "ICA", "BCI", and "EEG". According to application purposes, 22 selected studies were categorized into three classes: (1) artifact removal [16-19], (2) enhancement of SNR of task-related EEG signals [20-35], (3) selection of optimal electrodes $[36,37]$. In the applications of artifact removal and SNR enhancement, ICA was used to design spatial filters to remove task-irrelevant activities such as blinks and movement artifacts. The application of electrode selection aimed to reduce the number of electrodes needed in a BCI system based on the spatio-spectral characteristics of independent brain components. Table 1 lists details of these studies, including their application purpose, reference information, and types of BCI design. These studies cover most types of BCI designs including visual evoked potential (VEP), auditory evoked potential (AEP), P300 eventrelated potential (ERP), motor imagery, movement planning, mental tasks, and 
sleepiness/drowsiness monitoring. It is quite clear that most studies fall into the category of enhancing the SNR of task-related EEG signals. The method corresponding to each of the three categories is described in detail with example data in subsections below.

Table 1. Classification of ICA's applications in BCI studies.

\begin{tabular}{lll}
\hline Application Purpose & Study & BCI Type \\
\hline Removing EEG artifacts & Wang et al. [16] & Movement planning \\
& Halder et al. [17] & Motor imagery \\
Ghanbari et al.[18] & Motor imagery \\
& Papadelis et al. [19] & Sleepiness monitoring \\
Enhancing SNR of task- & Xu et al. [20] & P300 \\
related EEG signals & Serby et al. [21] & P300 \\
& Li et al. [22] & P300 \\
& Naeem et al. [23] & Motor imagery \\
& Delorme at al. [24] & Motor imagery \\
& Peterson [25] & Motor imagery \\
& Hung et al. [26] & Motor imagery \\
& Qin et al. [27] & Motor imagery \\
& Wang et al. [28] & Motor imagery \\
& Lee et al. [29] & VEP \\
& Hill et al. [30] & AEP \\
& Lin et al. [31] & Drowsiness monitoring \\
& Lan et al. [32] & Mental tasks \\
& Erfanian et al. [33] & Mental tasks \\
& Wang et al. [34] & Movement planning \\
& Hammon et al. [35] & Movement planning \\
& Wang et al. [36] & VEP \\
& Lou et al. [37] & Motor imagery \\
\hline
\end{tabular}

\subsection{Artifact removal}

EEG signals are often contaminated by pervasive artifacts such as blinks and motions. These artifacts might seriously deteriorate the system performance of BCIs [38]. To make a BCI system more robust, movement and other artifacts need to be eliminated before the task-related EEG features can be extracted for classification. The superiority of ICA in EEG artifact removal has been well demonstrated by many studies [39]. In this application category, ICA aims to separate and eliminate the artifact-related non-neural activities from the EEG signals.

Wang et al. used ICA to correct EEG signals recorded in a movement-planning task, which involved a lot of eye and muscle movements [16]. The EEG signals encoding movement directions can be applied to predict the direction of an intended movement (e.g., reach and saccade) after removing artifact components arising 
from eye and muscle activities. In motor imagery-based BCIs, system performance (e.g., classification accuracy or the R-square values of features) was improved after removing EOG/EMG artifacts $[17,18]$. In a drowsiness monitoring study [19], the ICA-based artifact removal was used as a routine approach to correct the EEG signals recorded in a driving task, which involved many head/body movements.

Fig. 2 illustrates the procedures of ICA-based artifact removal. In this example, the scalp EEG data recorded during reach/saccade planning and execution were contaminated by artifacts [16]. The artifact-removal method consists of three procedures: (1) apply ICA to scalp EEG data; (2) identify and remove the artifactrelated ICs; (3) project EEG-related ICs back to scalp electrodes to reconstruct artifact-corrected EEG data. In general, identification of artifact ICs can be performed using prior knowledge of spatio-temporal characteristics in EEG artifacts. For example, the IC corresponding to horizontal eye movement has a two-dipole distribution with opposite polarities over the bilateral prefrontal areas (see Fig. 1). As shown in Fig. 2, the SNR of the EEG signals has been considerably improved after removing the artifact ICs including EOG, ECG, and EMG. In practice, online implementation of this approach can effectively improve the robustness of an online BCI system.
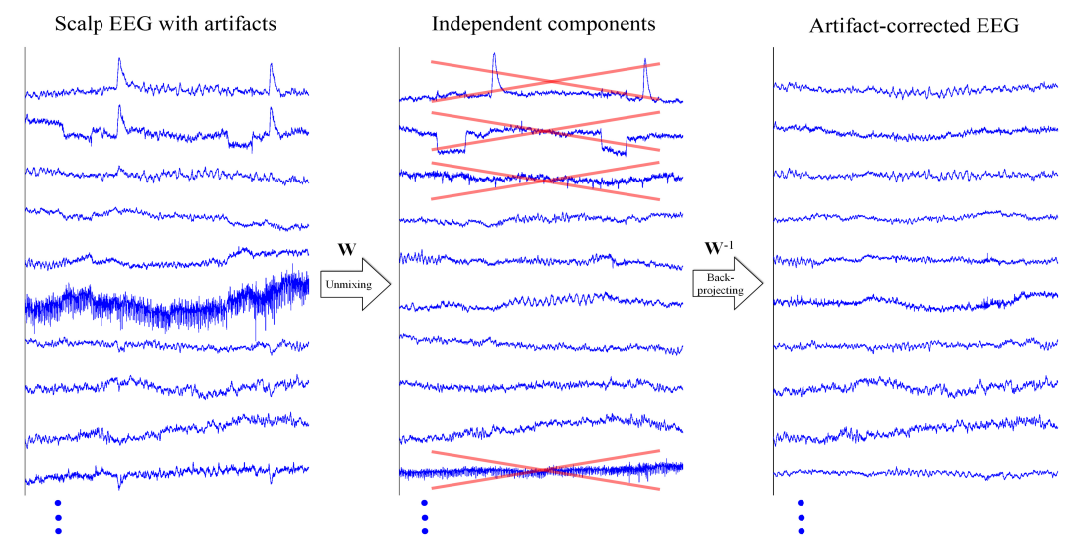

Fig. 2. Illustration of the ICA-based approach for artifact removal. Activities of ICs were obtained by applying ICA to scalp EEG data. Artifact (e.g., EOG, ECG, and EMG) ICs were identified and removed from the obtained ICs. Artifact-corrected EEG signals were obtained by only projecting the brain components back to the scalp electrodes.

\subsection{SNR enhancement of task-related EEG signals}

Spatial filtering is one of the most important signal processing techniques employed in BCIs using multichannel EEG [40]. The basic principle of spatial filter- 
ing is to eliminate task-irrelevant signals through linearly weighting different channels, and thus, enhances the SNR of task-related EEG signals. Many multidimensional data-processing methods have been adopted in recent BCI studies. For example, the common spatial pattern (CSP) method [41], the canonical correlation analysis (CCA) [42], and ICA, have been successfully applied to the motor imagery, the SSVEP, and the P300-based BCIs respectively. In general, the ICA-based spatial filtering method has two advantages: (1) it is an unsupervised learning method and therefore no labeled data are required; (2) it allows exploring the relationship between human behavior and the spatio-spectral pattern of an IC, facilitating the understanding of the specific neural mechanism. As listed in Table 1, the ICA-based spatial filtering has been widely applied to most types of BCIs including P300 [20-22], motor imagery [23-28], VEP [29], AEP [30], drowsiness monitoring [31], mental tasks [32, 33], and movement planning [34, 35]. Generally, these studies aimed to enhance the SNR of task-related EEG signals by ICA so that the system performance (e.g., classification accuracy) can be improved. In practice, only a small number of task-related EEG ICs will be selected for obtaining spatial filters according to their capabilities for discriminating different tasks.

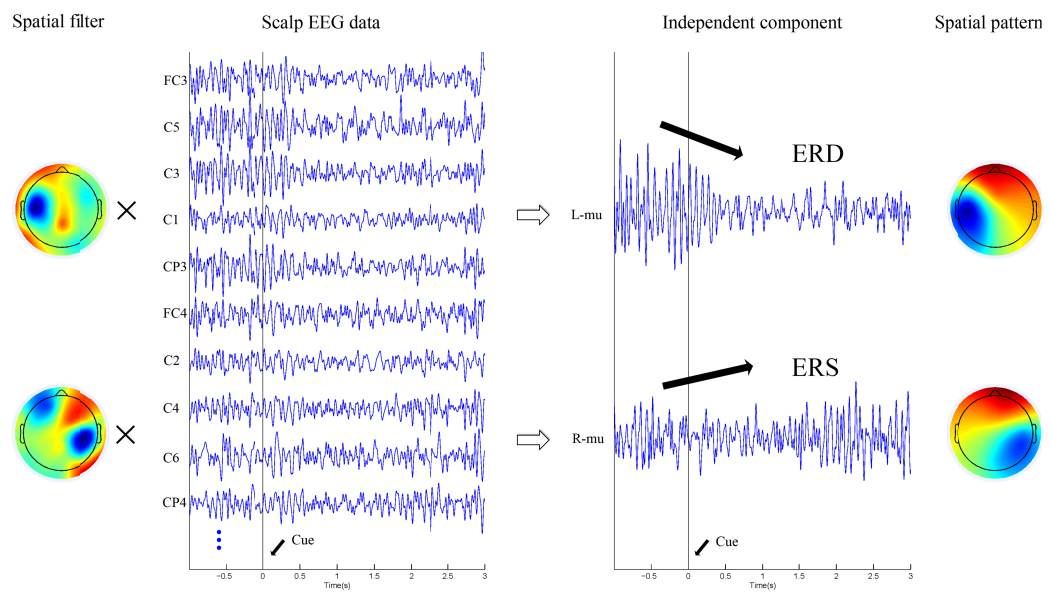

Fig. 3. ICA-based spatial filtering for extracting task-related brain activities during motor imagery. In this trial, the subject was instructed to imagine right-hand movement after a visual cue appeared at time 0 . After ICA, two ICs with characteristic spatio-sepectral patterns were selected as the motor components (L-mu and R-mu ICs), which were dominant by the mu rhythm in the frequency domain. The corresponding weighting vectors in the unmixing matrix (W) were selected to be used as spatial filters. Arrows indicate a contralateral ERD and an ipsilateral ERS, which can be more clearly observed in the IC activities than the scalp EEG channel data.

The major steps of the ICA-based spatial filtering approach include: (1) application of ICA to the training data; (2) identification of task-related ICs (i.e., the mu ICs in this case); and (3) the application of the corresponding spatial filters to EEG data before the training and testing steps of the classification process. Fig. 3 shows 
an example of applying ICA-based spatial filters to enhance motor activities in a motor imagery-based BCI. IC activities with higher SNR of motor activities than the scalp channel data can be obtained by multiplying motor-related spatial filters to the scalp EEG data. As shown in Fig. 3, the spatial filters have the largest positive weights over the sensorimotor areas on both hemispheres with some negative weights around this area functioning as linear combinations to eliminate common background activities. The spatial patterns corresponding to the mu ICs show very typical dipolar distributions over the sensorimotor areas, indicating the source locations of mu activity modulated by motor imagery. In this example, visually cued motor imagery of right hand movement induced a contralateral event-related desynchronization (ERD) and an ipsilateral event-related synchronization (ERS) of the mu rhythm (indicated by arrows in Fig. 3), which were more clearly shown in IC activities than the unprocessed scalp EEG channel data.

\subsection{Electrode selection}

An optimal selection of a small number of electrodes plays an important role in the design of a practical BCI system for real-life applications [6]. For example, in an SSVEP BCI, the goal of electrode selection is to achieve SSVEPs with a high SNR using a bipolar EEG channel consisting of a signal electrode and a reference electrode [36]. In practice, the electrode giving the strongest SSVEP, which is generally located in the occipital region, is selected as the signal electrode. The reference electrode is searched under the following criteria: its SSVEP should be weak, and its position should be close to the signal electrode so that its noise activity is similar to that of the signal electrode. In this way, a high SNR can be obtained with the bipolar channel because most of the spontaneous background activities are eliminated after the subtraction, while the SSVEP component is mostly retained.

Due to its superiority in decomposing independent brain sources, ICA can facilitate the electrode selection in BCIs. Wang et al. [36] developed an ICA-based approach for electrode selection in an SSVEP BCI. The detailed procedures are described as follows:

1) ICA decomposition. Thirteen-channel EEG signals $\boldsymbol{x}$ (with 13-Hz SSVEPs) between $\mathrm{Pz}$ and $\mathrm{Oz}$ (Fig. 4(a)) were selected as the input for ICA decomposition. Through ICA, 13 independent components were obtained as estimates of brain sources $\boldsymbol{s}$ including SSVEP components (signal) and other background EEG components (noise).

2) Reconstruction of signal and noise. The ICs with high SNR of SSVEP (i.e., the ratio of EEG power at $13 \mathrm{~Hz}$ to the EEG power in the rest of spectrum) were taken to be the true SSVEP-laden components and the remaining ICs were considered as background noise components. Through projecting the SSVEP sources and 
the noise sources back to the scalp electrodes, the SSVEP and noise activities at each electrode over the scalp can be separated.

3) Selection of the signal electrode. Power spectrum density (PSD) analysis was performed for calculating the SNR of the SSVEP. Fig. 4(a) shows PSDs of original channel data and decomposed SSVEP and noise activities on all 13 scalp electrodes. The electrode giving the strongest SSVEP activity (i.e., PO2) was selected as the signal channel.

4) Selection of the reference electrode. The correlation of the SSVEP activity and the noise activity between electrodes was calculated. The ratio of the SSVEP correlation to the noise correlation between other electrodes and the signal electrode is the criterion for selecting the reference electrode. Electrodes with high noise correlation and low SSVEP correlation are good candidates.

Fig. 4 shows an example of the proposed approach on one subject. As shown in Fig. 4(a), the SSVEP of this subject is highly contaminated by spontaneous EEG signals. It is difficult to choose a good bipolar channel from the original EEG channel data. Through ICA decomposition, the distribution of SSVEP activities shows that PO2 has the most significant SSVEP. As indicated by an arrow in Fig. 4(a), POz was selected as the reference channel due to its low SSVEP correlation and high noise correlation to PO2. Fig. 4(b) proves that the PO2-POz bipolar channel can significantly enhance the SNR of SSVEP due to the elimination of the common noise activities.

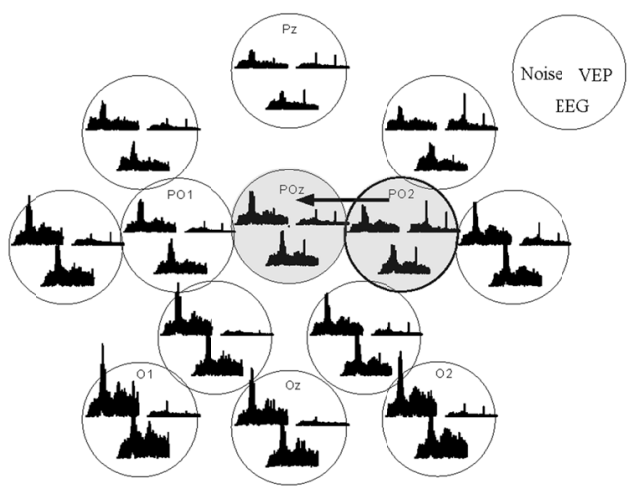

(a)

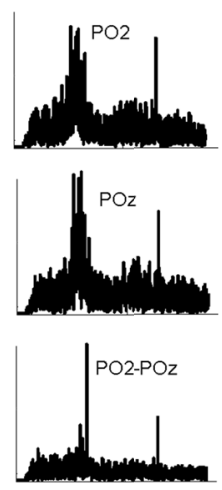

(b)

Fig. 4. (a) Illustration of the ICA-based approach for electrode selection in an SSVEP BCI. Thirteen electrodes around the occipital region were used in ICA to decompose EEG into SSVEP and background noise activities. For each channel, PSDs of scalp EEG, SSVEP and noise activities are put together for comparison. The arrow and shaded areas indicate the selected signal (PO2) and reference (POz) electrodes. (b) PSDs for monopolar channels PO2, POz, and the POz-PO2 bipolar channel.(adapted from [36] with permission from IEEE)

Not limited to the SSVEP-based BCI, this approach could be easily adapted to other BCI systems. For example, Lou et al. [37] developed a similar approach for 
optimizing bipolar electrodes in a motor imagery-based BCI. In their study, ICA was used for separating background alpha rhythms from the sensorimotor mu rhythms. Typical bipolar leads between the sensorimotor areas and the prefrontal areas (e.g., C3-FCz and $\mathrm{C} 4-\mathrm{FCz}$ ) were demonstrated most efficient for extracting the motor imagery induced power change of the mu rhythms.

\section{ICA-based zero-training BCI}

As mentioned above, EEG-based BCIs often use spatial filters to improve the SNR of task-related EEG activities [40]. To obtain robust spatial filters, large amounts of labeled data, which are often expensive and labor-intensive to obtain, need to be collected in a training procedure before online BCI control. Recently, several studies have developed zero-training methods using a session-to-session scenario in order to alleviate this problem [43]. To our knowledge, a state-to-state translation, which applies spatial filters derived from one state to another, has never been reported. This study proposes a state-to-state, zero-training method to construct spatial filters for extracting EEG changes induced by motor imagery. The unsupervised nature makes ICA a potential tool to obtain task-related spatial filters even from task-irrelevant data. In this study, ICA was separately applied to the multichannel EEG signals in the resting and the motor imagery states to obtain spatial filters specific for extracting the mu components. The resultant spatial filters were then applied to single-trial EEG to differentiate left- and right-hand imagery movements.

\subsection{Experiment and data recording}

Nine healthy right-handed volunteers (six males and three females, aged between 22 and 25) participated in the BCI experiments [44]. Fig. 5 shows the paradigm for online motor imagery-based BCI control with visual feedback. The left- and right-hand movement imaginations were designated to control vertical cursor movement on the screen. The subject sat comfortably in an armchair, facing a computer screen displaying visual feedback. The duration of each trial was 8 seconds. During the first 2 seconds, while the screen was blank, the subject was in the resting state. Immediately after these brief periods, a visual cue (arrow) was presented on the screen, indicating the imagery task to be performed. The arrows pointing upwards and downwards indicated the imagination of the left hand and the right hand movement, respectively. After 3 seconds, a cursor started to move at a constant speed from the left side to the right side of the screen. The vertical position of the cursor was determined by the power difference of mu rhythm between the left and right hemispheres (C3 and $\mathrm{C} 4$ electrodes). After 8 seconds, a 
true or false mark appeared on the screen to indicate the final result of the trial and the subject was asked to relax and wait for the next task.

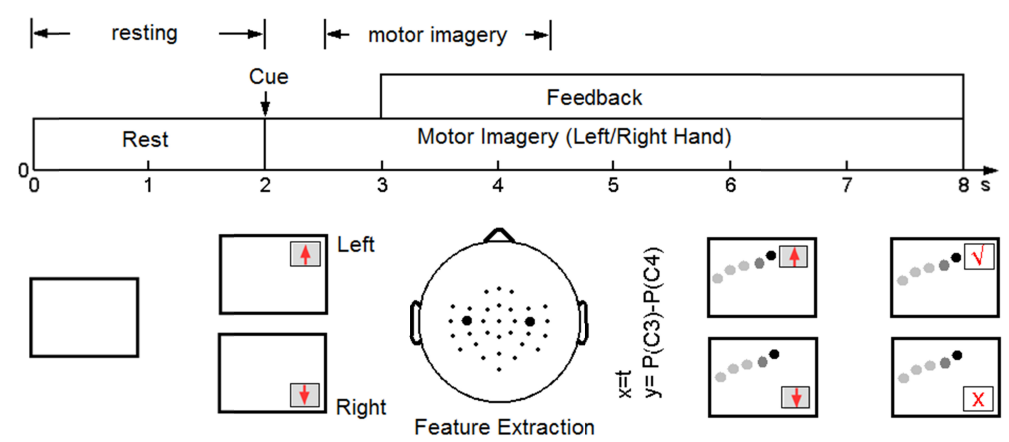

Fig. 5. Experiment paradigm for the motor imagery-based brain-computer interface.

32-channel EEG signals referenced to the CMS-DRL ground were recorded using a BioSemi ActiveTwo system with the electrodes placed according to the 1020 international system. The signals were digitized at $256 \mathrm{~Hz}$ and band-pass filtered $(2-30 \mathrm{~Hz})$ for further analysis. For each subject, the experiment consisted of four blocks, each including 60 trials (30 trials per class). There were 3-5 minutes of breaks between two consecutive blocks. A total of 240 trials (120 trials per class) were recorded for each subject.

\subsection{Method}

\subsubsection{ICA decomposition}

As indicated in Fig. 5, the $0-2 \mathrm{~s}$ and 2.5-4.5s segments in a trial were selected to represent the resting state and the motor imagery state, respectively. For each subject, ICA was performed on data under the two states separately. For each state, data of all trials were concatenated to a 480 -second (240 trials $\times 2$ seconds) long data segment. Because the size of data was very limited (480 seconds), to improve the robustness of ICA, 32-channel data were first projected to a 15-dimensional subspace using principal component analysis (PCA). Then, for each subject, ICA resulted in two sets of $15 \times 32$ spatial filters $\left(\mathbf{W}_{\text {rest }}\right.$ and $\left.\mathbf{W}_{\mathrm{mi}}\right)$ and $32 \times 15$ spatial projections ( $\mathbf{W}_{\text {rest }}^{-1}$ and $\mathbf{W}_{\mathrm{mi}}^{-1}$ ) corresponding to the resting and motor imagery.

\subsubsection{ICA-based spatial filters}


In previous studies, ICA has shown its robustness in finding motor components, which have characteristic features in spatial and frequency domains [37]. This study used two criteria to identify the motor components: (1) the spatial pattern, which suggests the source location of the component, should be consistent with the scalp projection of the sensorimotor cortex on each hemisphere; (2) the PSD of the component should match the typical spectral profile of the mu/beta rhythms. In practice, a motor component should fit both criteria. After identifying the two motor ICs, the corresponding weighting vectors in the unmixing matrix $(\mathbf{W})$ were used as spatial filters for enhancing the sensorimotor mu/beta rhythms.

\subsubsection{Resting-to-work translation}

Suppose the two motor components in the resting state and the motor imagery state have strong similarities, it might be feasible to use the spatial filters obtained from the data in the resting state as estimates of the spatial filters for the motor imagery state. The proposed method can be described as follows:

$$
\widehat{\mathbf{W}}_{\text {motor_mi }}^{-1}=\mathbf{W}_{\text {motor_rest }}^{-1} \quad \widehat{\mathbf{W}}_{\text {motor_mi }}=\mathbf{W}_{\text {motor_rest }}
$$

where $\mathbf{W}_{\text {motor_rest }}$ and $\mathbf{W}_{\text {motor_mi }}$ are motor-related spatial filters for the resting state and the motor imagery state respectively. Fig. 6 illustrates the principle of the proposed method. In this paradigm, data in the resting state, which do not require the subject's attention or action, and the motor imagery state were totally nonoverlapped. The spatial filters derived from the resting data were estimates of the spatial filters for the motor imagery data. In practice, the resting EEG data can be easily collected before a BCI session.

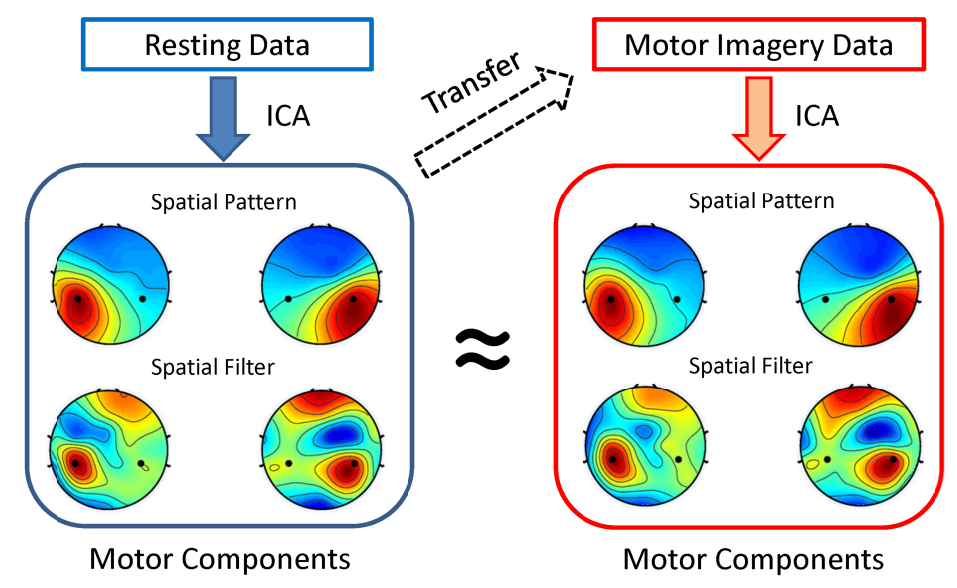

Fig. 6. Diagram of translating spatial filters from the resting state to the motor imagery state. 


\subsubsection{Feature extraction and classification}

This study compares the classification performance of motor-imagery BCIs based on band-pass $(8-30 \mathrm{~Hz})$ power of the mu and beta rhythms extracted using four methods: (1) monopolar C3 and C4 electrodes; (2) spatial filtering based on ICA using the resting data; (3) spatial filtering based on ICA using the motor imagery data; (4) CSP-based spatial filtering. After feature extraction, Fisher discriminant analysis (FDA) [45] was used to discriminate left and right hand motor imagery. The two-dimensional feature vector, which represented EEG power over the motor areas of two hemispheres, was fed into the FDA classifier for identifying the imagined hand. A 10x10-fold cross-validation was used to estimate the classification accuracy for each subject.

\subsection{Results}

Fig. 7 shows spatial patterns of the motor components in the resting state and the motor imagery state for all subjects. All the components show a typical dipolarlike topography, which is widespread over the sensorimotor cortex on left or right hemisphere of the brain, and shows the highest amplitudes at $\mathrm{C} 3$ and $\mathrm{C} 4$ electrodes. To quantitatively evaluate the topographical similarity, this study calculated the correlations of spatial patterns of the motor components between the two states for each subject. The correlations were obtained by computing correlation coefficients of the $1 \times 32$ vectors. Spatial patterns (i.e. projections of the components to the scalp) between the resting and the motor imagery states were very comparable (mean correlation coefficients of $0.95 \pm 0.05$ and $0.94 \pm 0.06$ for left and right ICs) for all subjects.

The FDA classifier used the four different types of EEG features as inputs to classify single-trial motor-imagery movements. Table 1 summarizes the results of 10x10-fold cross-validation. A paired t-test across subjects was used to test the statistical significance of the differences between different feature extraction methods. As expected, compared to the monopolar method, all spatial-filtering methods achieved significantly higher classification accuracies $(87.0 \%, 85.9 \%$, and $86.4 \%$ vs. $80.4 \%, p<0.01)$. The results of ICA trained with the motor imagery data were slightly better than those trained with the resting data $(87.0 \%$ vs. $85.9 \%)$, but the difference was not statistically significant $(\mathrm{p}>0.1)$. The results of using CSP-filtered (based on motor-imagery data) were comparable with those using ICA trained with motor imagery data $(86.4 \%$ vs. $87.0 \%, \mathrm{p}>0.1)$ and resting data $(86.4 \%$ vs. $85.9 \%, \mathrm{p}>0.1)$. These findings demonstrated the effectiveness of translating resting spatial filters to classifying motor imagery EEG data using ICA. 

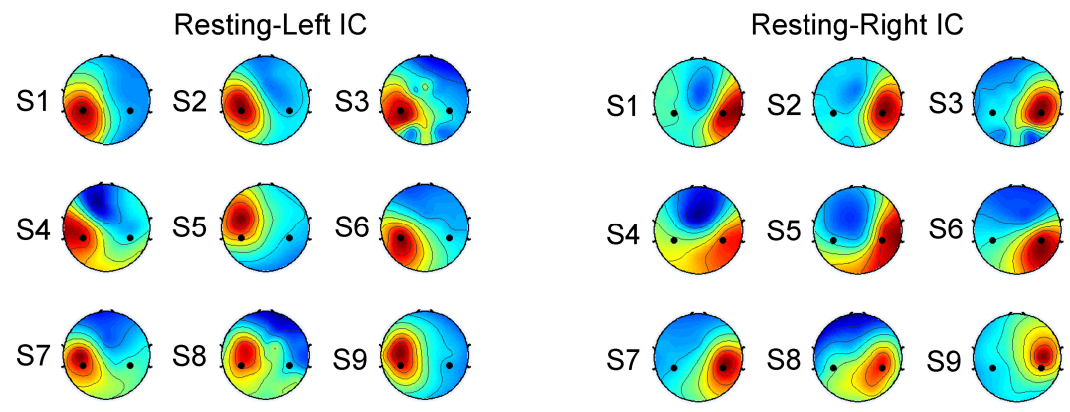

(a)
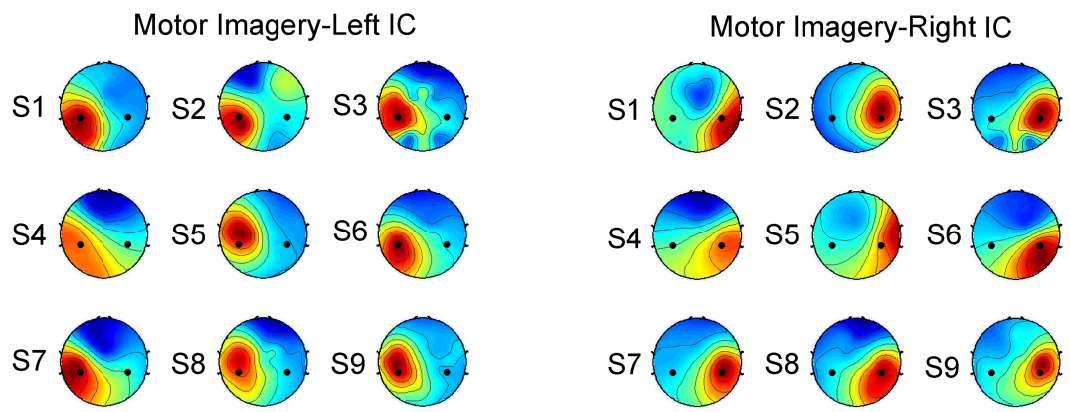

(b)

Fig. 7. Spatial patterns of the left and right motor components for all nine subjects (S1 to S9): (a) spatial patterns of the resting state (left panels: left motor IC on the left hemisphere, right panels: right motor IC on the right hemisphere); (b) spatial patterns of the motor imagery state.

Table 2. Classification accuracy (\%) for all subjects using different feature extraction methods.

\begin{tabular}{ccccc}
\hline & \multicolumn{4}{c}{ Method } \\
\cline { 2 - 5 } Subjects & Monopolar & ICA-mi & ICA-rest & CSP \\
S1 & 86 & 84 & 84 & 88 \\
S2 & 66 & 70 & 70 & 72 \\
S3 & 84 & 92 & 92 & 90 \\
S4 & 86 & 94 & 88 & 93 \\
S5 & 84 & 90 & 88 & 88 \\
S6 & 93 & 96 & 96 & 92 \\
S7 & 87 & 92 & 93 & 92 \\
S8 & 85 & 97 & 95 & 95 \\
S9 & 53 & 67 & 68 & 69 \\
Mean & $80.4 \pm 12$ & $87.0 \pm 9$ & $85.9 \pm 11$ & $86.4 \pm 9$ \\
\hline
\end{tabular}




\section{Conclusion and discussions}

This chapter presents an up-to-date literature review on ICA in BCI applications by categorizing related studies into three classes (i.e., artifact removal, SNR enhancement of task-related EEG signals, and electrode selection) according to the roles of ICA. The basic principles and methodologies behind these applications have been fully illustrated through examples with real EEG data. This chapter also describes an extended application of the ICA-based spatial filter in the development of a zero-training method for a motor imagery-based BCI. In summary, this chapter shows that ICA can make a substantial contribution to the practical design of BCI systems.

Although the advantages of using ICA in EEG-based BCIs have been clearly shown in this chapter, most applications were developed and demonstrated only with offline data analysis. Among all examples presented in Table 1, only three studies performed online system implementation ([17], [21], and [29]). The study in [17] implemented an online-automated artifact removal technique for BCI using ICA. The P300-based BCI system developed in [21] adapted ICA-based filters obtained in previous offline sessions to current online sessions to enhance the P300 potentials. The VEP-based BCI system developed in [29] used predefined spatial templates to select VEP-related ICs after preforming ICA in near real time. Although these studies showed some functionality of online implementation of ICA in BCI systems, possibilities and practicalities of this approach still need further investigation.

Currently, researchers still face some technical challenges to truly implement ICA in online BCI systems. First, hardware and software must meet the computational requirements of ICA. In some situations, due to EEG nonstationarity [46], the ICA processing might need to be performed in near real time. Under the circumstances, adaptive algorithms can be used to reduce the computational complexity of ICA. Furthermore, the recent demand of mobile and wearable BCI systems poses more stringent limitations on their computational performance. A system-on-chip design [47] might be a practical solution to this problem. Second, automatic methods for identifying task relevant ICs need to be developed. In most studies, the IC identification was performed manually based on researchers' personal experiences. In real-time applications, this procedure will be labor-intensive and time-consuming, and therefore, decrease the system's practicality. Pattern recognition methods might be employed to realize automatic IC identification by comprehensively considering ICs' properties in time, frequency, and spatial domains. Third, stability and robustness of ICA based spatial filters always depend on the amount of training data. In an online BCI application, more training data require a longer user training time, thereby reducing the practicality of the BCI system. To alleviate this problem, a session-to-session translation, as well as the state-to-state translation method proposed in this chapter, might be a practical so- 
lution. Taken together, by solving these technical issues using advanced platform, signal processing, and machine learning techniques, ICA could make a substantial contribution to the development of practical online BCI systems.

Acknowledgments This work was supported by a gift fund from Abraxis Bioscience Inc. Research was also sponsored in part by Office of Naval Research, Army Research Office (under contract number W911NF-09-1-0510) and Army Research Laboratory (under Cooperative Agreement Number W911NF-10-2-0022). The views and the conclusions contained in this document are those of the authors and should not be interpreted as representing the official policies, either expressed or implied, of the Army Research Office, Army Research Laboratory, Office of Naval Research, or the U.S Government. The U.S Government is authorized to reproduce and distribute reprints for Government purposes notwithstanding any copyright notation herein. Melody Jung is appreciated for editorial assistance.

\section{References}

[1] Wolpaw JR, Birbaumer N, McFarland DJ, Pfurtscheller G, Vaughan TM (2002) Braincomputer interfaces for communication and control. Clin Neurophysiol 113: 767-791.

[2] Lebedev MA, Nicolelis MAL (2006) Brain-machine interfaces: past, present and future. Trends Neurosci 29: 536-546.

[3] Birbaumer N (2006) Brain-computer-interface research: Coming of age. Clin Neurophysiol 117: 479-483.

[4] Wang Y, Gao X, Hong B, Jia C, Gao S (2008) Brain-computer interfaces based on visual evoked potentials: feasibility of practical system designs. IEEE EMB Mag 27: 64-71.

[5] P Brunner, L Bianci, C Guger, F Cincotti, and G Schalk (2011) Current trends in hardware and software for brain-computer interfaces (BCIs). J Neural Eng 8: 025001.

[6] Wang Y, Gao X, Hong B, Gao S (2010) Practical designs of brain-computer interfaces based on the modulation of EEG rhythms. In: Graimann B, Pfurtscheller G (ed) Invasive and NonInvasive Brain-Computer Interfaces. Springer, Berlin.

[7] Lotte F, Congedo M, Lecuyer A, Lamarche F, Arnaldi B (2007) A review of classification algorithms for EEG-based brain-computer interfaces. J Neural Eng 4: R1-R13.

[8] Lee TW, Girolami M, Sejnowski TJ (1999) Independent component analysis using an extended infomax algorithm for mixed subgaussian and supergaussian sources. Neural Comput 11: 417-441.

[9] Delorme A, Makeig S (2004) EEGLAB: an open source toolbox for analysis of single-trial EEG dynamics including independent component analysis. J Neurosci Meth 134: 9-21.

[10] Hyvärinen A, Oja E (2000) Independent component analysis: algorithms and application. Neural Networks 13: 411-430.

[11] James CJ, Hesse CW (2005) Independent component analysis for biomedical signals. Physiol Meas 26: R15-R39.

[12] Vigario R, Sarela J, Jousmiki V, Hamalainen M, Oja E (2000) Independent component approach to the analysis of EEG and MEG recordings. IEEE Trans Biomed Eng 47: 589-593.

[13] Jung TP, Makeig S, McKeown MJ, Bell AJ, Lee TW, Sejnowski TJ (2001) Imaging brain dynamics using independent component analysis. Proc IEEE 89: 1107-1122.

[14] Makeig S, Westerfield M, Jung TP, Townsend J, Courchesne E, Sejnowski T J (2002) Dynamic brain sources of visual evoked responses. Science 295: 690-694.

[15] Kachenoura A, Albera L, Senhadji L, Comon P (2008) ICA: a potential tool for bci systems. IEEE Signal Processing Mag 25: 57-68. 
[16] Wang Y, Jung TP (2011) A collaborative brain-computer interface for improving human performance. PLoS ONE 6: e20422.

[17] Halder S, Bensch M, Mellinger J, Bogdan M, Kubler A, Birbaumer N, Rosenstiel W (2007) Online artifact removal for brain-computer interfaces using support vector machines and blind source separation. Comput Intell Neurosci, 2007: 82069.

[18] Asadi Ghanbari A, Nazari Kousarrizi MR, Teshnehlab M, Aliyari M (2009) An evolutionary artifact rejection method for brain computer interface using ICA. Int J Elec Comput Sci 9: 461-466.

[19] Papadelis C, Chen X, Kourtidou-Papadeli C, Bamidis PD, Chouvarda I, Bekiaris E, Maglaveras N (2007) Monitoring sleepiness with on-board electrophysiological recordings for preventing sleep-deprived traffic accidents. Clin Neurophysiol 118: 1906-1922.

[20] Xu N, Gao X, Hong B, Miao X, Gao S, Yang F (2004) BCI competition 2003-Data set IIb: enhancing P300 wave detection using ICA-based subspace projections for BCI applications. IEEE Trans Biomed Eng 51: 1067-1072.

[21] Serby H, Yom-Tov E, Inbar GF (2005) An improved P300-based brain-computer interface. IEEE Trans Neural Syst Rehabil Eng 13: 89-98.

[22] Li K, Sankar R, Arbel Y, Donchin E (2009) Single trial independent component analysis for P300 BCI system. Proc 31th Int IEEE EMBS Conf, Minneapolis, USA, pp. 4035-4038.

[23] Naeem M, Brunner C, Leeb R, Graimann B, Pfurtscheller G (2006) Seperability of fourclass motor imagery data using independent components analysis. J Neural Eng 3: 208-216.

[24] Delorme A, Makeig S (2003) EEG changes accompanying learned regulation of 12-Hz EEG activity. IEEE Trans Neural Syst Rehabil Eng 11: 133-137.

[25] D. A. Peterson (2005) Feature selection and blind source separation in an EEG-based BrainComputer Interface. EURASIP J Applied Signal Processing 19: 3128-3140.

[26] Hung CI, Lee PL, Wu YT, Chen LF, Yeh TC, Hsieh JC (2005) Recognition of motor imagery electroencephalography using independent component analysis and machine classifiers. Ann Biomed Eng 33: 1053-70.

[27] Qin L, Ding L, He B (2004) Motor imagery classification by means of source analysis for brain-computer interface applications. J Neural Eng 1: 135-141.

[28] Wang S, James CJ (2007) Extracting rhythmic brain activity for brain-computer interfacing through constrained independent component analysis. Comput Intell Neurosci 2007: 41468.

[29] Lee PL, Hsieh JC, Wu CH, Shyu KK, Chen SS, Yeh TC, Wu YT (2006) The brain computer interface using flash visual evoked potential and independent component analysis. Ann Biomed Eng 34: 1641-1654.

[30] Hill NJ, Lal TN, Bierig K, Birbaumer N, Scholkopf B (2004) Attentional modulation of auditory event-related potentials in a brain-computer interface. Proc IEEE International Workshop on Biomedical Circuits and Systems, Singapore, pp. 17-19.

[31] Lin CT, Wu RC, Liang SF, Chao WH, Chen YJ, Jung TP (2005) EEG-based drowsiness estimation for safety driving using independent component analysis. IEEE Trans Circuits and Systems I 52: 2726-2738.

[32] Tian L, Erdogmus D, Adami A, Pavel M (2005) Feature selection by independent component analysis and mutual information maximization in EEG signal classification. Proc 2005 IEEE International Joint Conference on Neural Networks, Montreal, Canada, pp. 3011-3016.

[33] Erfanian A, Erfani A (2004) ICA-based classification scheme for EEG-based braincomputer interface: the role of mental practice and concentration skills. Proc 26th Int IEEE EMBS Conf, San Francisco, USA, pp.235-238.

[34] Wang Y, Makeig S (2009) Predicting intended movement direction using EEG from human posterior parietal cortex In: Schmorrow DD, Estabrooke IV, Grootjen M (ed) Foundations of augmented cognition: Neuroergonomics and operational neuroscience (HCII 2009). Springer, Berlin. pp 437-446.

[35] Hammon PS, Makeig S, Poizner H, Todorov E, de Sa VR (2008) Predicting reaching targets from human EEG. IEEE Signal Processing Mag 25: 69-77. 
[36] Wang Y, Wang R, Gao X, Hong B, Gao S (2006) A practical VEP-based brain-computer interface. IEEE Trans Neural Syst Rehabil Eng, 14: 234-239.

[37] Lou B, Hong B, Gao X, Gao S (2008) Bipolar electrode selection for a motor imagery based brain-computer interface. J Neural Eng 5: 342-349.

[38] M. Fatourechi, A. Bashashati, R. Ward, and G. Birch (2007) EMG and EOG artifacts in brain computer interface systems: A survey. Clin Neurophysiol 118: 480-494.

[39] Jung TP, Makeig S, Humphries C, Lee TW, McKeown MJ, Iragui V, Sejnowski TJ (2000) Removing electroencephalographic artifacts by blind source separation. Psychophysiology 37: 163-78.

[40] McFarland DJ, McCane LM, David SV, Wolpaw JR (1997) Spatial filter selection for EEGbased communication. Electroenceph Clin Neurophysiol 103: 386-394.

[41] Blankertz B, Tomioka R, Lemm S, Kawanabe M, Müller KR (2008) Optimizing spatial filters for robust EEG single-trial analysis. IEEE Signal Processing Mag 25: 41-56.

[42] Bin G, Gao X, Yan Z, Hong B, Gao S (2009) An online multi-channel SSVEP-based braincomputer interface using a canonical correlation analysis method. J Neural Eng 6: 046002.

[43] Krauledat M, Tangermann M, Blankertz B, Müller KR (2008) Towards zero training for brain-computer interfacing. PLoS ONE 3: e2967.

[44] Wei Q, Wang Y, Gao X, Gao S (2007) Amplitude and phase coupling measures for feature extraction in an EEG-based brain-computer interface. J Neural Eng 4: 120-129.

[45] Duda RO, Hart PE, Stork DG (2000) Pattern Classification (2nd Edition). Wiley Interscience Press, New York.

[46] Krauledat M (2008) Analysis of nonstationarities in EEG signals for improving braincomputer interface performance. PhD thesis, Technische Universität Berlin, Fakultät IVElektrotechnik und Informatik.

[47] Fang WC, Chen CK, Chua E, Fu CC, Tseng SY, Kang S (2010) A low power biomedical signal processing system-on-chip design for portable brain-heart monitoring systems. Proc 2010 Int Conf Green Circuits and Systems (ICGCS), Shanghai, China, pp.18-23.

Abstract In the past two decades, independent component analysis (ICA) has been widely used for Electroencephalogram (EEG) signal processing. Recently, towards real-life applications, the EEG-based brain-computer interface (BCI) research has posed a demand for advanced signal processing techniques such as ICA. This chapter focuses on using the ICA-based approaches to improve performance and practicality of BCI systems. This chapter first presents an up-to-date literature review on the applications of ICA in EEG-based BCIs. According to application purposes, these studies are divided into three categories: (1) artifact removal; (2) enhancement of signal-to-noise ratio (SNR) of task-related EEG signals; (3) selection of optimal electrodes. The methods will be described in detail with example data used in BCIs. Next, this chapter reports our recent progress on an ICA-based zero-training (i.e., resting-to-work translating) BCI, which aims to facilitate user training for a motor imagery BCI. 